\title{
Neonatal Bacillus Calmette-Guérin vaccination alleviates lipopolysaccharide-induced neurobehavioral impairments and neuroinflammation in adult mice
}

\author{
JUNHUA YANG ${ }^{*}$, FANGFANG QI ${ }^{*}$ and ZHIBIN YAO \\ Department of Anatomy and Neurobiology, Zhongshan School of Medicine, \\ Sun Yat-Sen University, Guangzhou, Guangdong 510080, P.R. China
}

Received August 21, 2015; Accepted April 29, 2016

DOI: $10.3892 / \mathrm{mmr} .2016 .5425$

\begin{abstract}
The Bacillus Calmette-Guérin (BCG) vaccine is routinely administered to human neonates worldwide. BCG has recently been identified as a neuroprotective immune mediator in several neuropathological conditions, exerting neuroprotection in a mouse model of Parkinson's disease and slowing the progression of clinically isolated syndrome in patients with multiple sclerosis. The immune system is significantly involved in brain development, and several types of neonatal immune activations exert influences on the brain and behavior following a secondary immune challenge in adulthood. However, whether the neonatal BCG vaccination affects the brain in adulthood remains to be elucidated. In the present study, newborn C57BL/6 mice were injected subcutaneously with BCG ( $10^{5}$ colony forming units) or phosphate-buffered saline (PBS). A total of 12 weeks later,
\end{abstract}

Correspondence to: Professor Zhibin Yao, Department of Anatomy and Neurobiology, Zhongshan School of Medicine, Sun Yat-Sen University, 74 Zhongshan No. 2 Road, Guangzhou, Guangdong 510080, P.R. China

E-mail: yao.zb@163.com

*Contributed equally

Abbreviations: 5-HT, 5-hydroxytryptamine; 5-HIAA, 5-hydroxyindoleacetic acid; BCG, Bacillus Calmette-Guérin; BDNF, brain-derived neurotrophic factor; BrdU, 5-bromo-2-deoxyuridine; $\mathrm{CFU}$, colony forming units; CNS, central nervous system; CON, control; DCs, dendritic cells; DG, dentate gyrus; FST, forced swimming test; HPA, hypothalamic-pituitary-adrenal; IFN- $\gamma$, interferon- $\gamma$; IGF-1, insulin-like growth factor-1; IL-1 $\beta$, interleukin-1 $\beta$; IL-4, interleukin-4; IL-6, interleukin-6; LPS, lipopolysaccharide; OFT, open field test; PBS, phosphate-buffered saline; ANOVA, analysis of variance; SEB, social exploratory behavior; SYSU, Sun Yat-Sen University; TNF- $\alpha$, tumor necrosis factor- $\alpha$; Tregs, regulatory T cells; TST, tail suspension test

Key words: immunization, sickness-like behavior, depression, cytokine, neurotrophin the mice were injected intraperitoneally with $330 \mu \mathrm{g} / \mathrm{kg}$ lipopolysaccharide (LPS) or PBS. The present study reported that the neonatal BCG vaccination alleviated sickness, anxiety and depression-like behavior, lessened the impairments in hippocampal cell proliferation and downregulated the proinflammatory responses in the serum and brain that were induced by the adult LPS challenge. However, BCG vaccination alone had no evident influence on the brain and behavior in adulthood. In conclusion, the neonatal BCG vaccination alleviated the neurobehavioral impairments and neuroinflammation induced by LPS exposure in adult mice, suggesting a potential neuroprotective role of the neonatal $B C G$ vaccination in adulthood.

\section{Introduction}

Bacillus Calmette-Guérin (BCG), a vaccine incorporated into routine childhood vaccination schedules globally, is administered to neonates and children worldwide (1). In addition to the prevention of tuberculosis, BCG has been used as an immune modulator and even as immunotherapy in certain non-infectious diseases (2-4). Furthermore, recent clinical and experimental studies have revealed that BCG has a neuroprotective role in several central nervous system (CNS) pathological conditions, including clinically isolated syndrome, multiple sclerosis, Parkinson's disease and experimental autoimmune encephalomyelitis (5-7).

Early-life immune activation has been well-established to regulate the programming of brain development and influence behavior in later life, predominantly using three animal models of early-life immune activation: Polycytidylic acid, Escherichia coli and lipopolysaccharide (LPS) $(8,9)$. However, whether neonatal BCG vaccination as immune pre-conditioning has consequences in the CNS, and its function in adulthood, remains to be elucidated.

There are two patterns through which early-life immune activation may affect behavior in later life (10). In one pattern, early-life immune activation is able to directly modulate or disrupt development $(11,12)$. In the other pattern, early-life immune activation can influence the immune system and alter the neuroimmune response to a subsequent immune challenge in adulthood $(13,14)$. Therefore, direct and indirect later-life 
effects on the brain and behavior were assessed in the present study.

Adult LPS administration has been demonstrated to induce behavior impairments and neuroinflammation in rodents $(15,16)$, and LPS has also been used for adult immune challenge in early-life-infected rodents $(17,18)$. Therefore, LPS injection was used as an adult immune challenge in the present study to investigate the potential indirect effects of neonatal $\mathrm{BCG}$ vaccination.

Sickness, depression and anxiety-like behaviors were observed within $24 \mathrm{~h}$ following LPS injection. Additionally, certain molecules associated with behavior and immune activation were examined, including cytokines, neurotrophins, 5-hydroxytryptamine (5-HT) and corticosterone, in the brain and/or serum.

\section{Materials and methods}

Animals and study design. The present study was approved by the Sun Yat-Sen University (SYSU) Institute Research Ethics Committee (Guangzhou, China) and was strictly performed according to the UK Animals (Scientific Procedures) Act, 1986 (19). Newborn litters of C57BL/6 mice were obtained from the SYSU Laboratory Animal Center (Guangzhou, China) and were reared under specific pathogen-free conditions. The mice were housed at a temperature of $25^{\circ} \mathrm{C}$ in $60 \%$ relative humidity on a 12-h light/dark cycle (lights on between 6:00 AM and 6:00 PM), and allowed free access to food and water. A total of four groups were used in the study: BCG/LPS, $\mathrm{BCG}$ alone, LPS alone and control $(\mathrm{CON})$ groups. The mice were administered with BCG (BCG/LPS and BCG groups) or PBS (LPS and CON groups) at birth. At 12 weeks old, the mice were administered LPS (BCG/LPS and LPS groups) or PBS (BCG and CON groups).

For each of the four behavioral tests, social exploratory behavior test (SEB), open field test (OFT), forced swimming test (FST) and tail suspension test (TST), a total of 16 newborn litters of C57BL/6 mice were used and 64 male pups were selected (4 pups/litter). The pups from each litter were distributed randomly into the 4 groups (1 pup/litter/group; total 16 pups/group). For bodyweight and food intake measurements, a total of 10 newborn litters were used and 40 male pups were selected (4 pups/litter). The pups from each litter were distributed randomly into the 4 groups (1 pup/litter/group; total $10 \mathrm{pups} /$ group). For the remaining experiments, a total of 6 newborn litters were used and 24 male pups were selected (4 pups/litter). The pups from each litter were distributed randomly into the 4 groups (1 pup/litter/group; total 6 pups/group). Pups were weaned at 3 weeks old. In the SEB paradigm, 4-week-old male juvenile conspecifics were used.

Neonatal immunization and adult immune challenge. Freeze-dried living BCG (D2-BP302 strain; Biological Institute of Shanghai, Shanghai, China) was dissolved in PBS. BCG was administered to mice at birth, imitating the age at which vaccination is performed in human infants. Each newborn mouse in the BCG/LPS and BCG groups was injected subcutaneously in the back with $25 \mu \mathrm{l} /$ mouse of BCG suspension containing $10^{5}$ colony forming units (CFU), as previously described (20); mice in the LPS and CON groups were injected with PBS in an identical manner. At 12 weeks old, each mouse in the BCG/LPS and LPS groups was injected with $0.33 \mathrm{mg} / \mathrm{kg}$ LPS (Sigma-Aldrich, St. Louis, MO, USA) intraperitoneally, and mice in the BCG and CON groups were injected with PBS in an identical manner. This dosage of LPS induces a proinflammatory cytokine response in the peripheral nervous system and brain, resulting in mild transient sickness behavior in adult mice (15).

SEB test. The mice were subjected to the SEB test 30 min prior to, and 4, 8 and $24 \mathrm{~h}$ following, LPS injection. The test was performed as described previously (15). To assess the motivation to perform SEB, a novel juvenile conspecific was placed into the home cage of the test subject for $10 \mathrm{~min}$. Behavior was videotaped and the total time spent by the test subject in social investigation (including anogenital sniffing and trailing) was calculated from the video records by a trained observer in a blinded manner. SEB was represented as the quantity of time spent by the test subject investigating the juvenile.

Body weight and food intake measurement. Body weight alteration and food intake of mice within the $24 \mathrm{~h}$ following LPS injection were calculated from body weight and food weight measured immediately prior to and $24 \mathrm{~h}$ following the LPS injection. Food intake was estimated according to a previously described method (21).

OFT. The OFT tests were performed $2.5 \mathrm{~h}$ following the LPS injection. This time interval was selected based on a previous study by Wang et al (22). The animals were individually placed in a plexiglass cubicle $(40 \times 40 \times 38 \mathrm{~cm})$. The spontaneous locomotor activity for each animal was recorded for $30 \mathrm{~min}$ by the Flex-Field activity system (San Diego Instruments, San Diego, CA, USA). The number of beam breaks by each mouse were counted automatically by the system. The apparatus was thoroughly cleaned with $70 \%$ ethanol following each trial.

FST. The mice were subjected to the FST task 30 min prior to, and 4, 8 and 24 h following, LPS injection. The animals were individually forced to swim for $6 \mathrm{~min}$ in an open cylindrical container (diameter, $10 \mathrm{~cm}$; height, $30 \mathrm{~cm}$ ), containing $20 \mathrm{~cm}$ of water (depth) at $22 \pm 1^{\circ} \mathrm{C}$. The total duration of immobility was recorded during the final 5-min period and was analyzed by a video tracking system EthoVision (Noldus Information Technology B.V., Wageningen, Netherlands).

TST. The mice were subjected to the TST task 30 min prior to, and 4, 8, and $24 \mathrm{~h}$ following, LPS injection. In a soundproof room, each mouse was suspended upside down by their tails for $6 \mathrm{~min}$. The total duration of immobility was measured during the final $5 \mathrm{~min}$ period.

5-Bromo-2-deoxyuridine (BrdU) labeling and tissue preparation. Together with LPS (in BCG/LPS and LPS mice) or PBS (in BCG and CON mice) injection, one dose of BrdU (50 mg/kg; Sigma-Aldrich, St. Louis, MO, USA) was injected into the mice. A repeat dose of BrdU was administered $12 \mathrm{~h}$ later. The mice were anesthetized with $10 \%$ chloral hydrate $(0.3 \mathrm{ml}$ per mouse, i.p.; Melone Pharmaceutical Co., Ltd., Dalian, China) and perfused transcardially with $4 \%$ paraformaldehyde a total 
of $24 \mathrm{~h}$ following the initial BrdU injection. The brains were excised and subsequently fixed overnight in $4 \%$ paraformaldehyde at $4^{\circ} \mathrm{C}$, and dehydrated with $30 \%$ sucrose at $4^{\circ} \mathrm{C}$ for $72 \mathrm{~h}$. Then, following freezing at $-20^{\circ} \mathrm{C}$, the brains were sliced into serial coronal sections $(40 \mu \mathrm{m})$ on a freezing microtome (Leica SM2000 R; Leica Microsystems GmBH, Wetzlar, Germany). Serial coronal sections $(40 \mu \mathrm{m})$ were collected and were stained for $\mathrm{BrdU}^{+}$cells.

Immunofluorescence and cell quantification. Specimens were incubated in 2- $\mathrm{N} \mathrm{HCl}$ for $30 \mathrm{~min}$ at $37^{\circ} \mathrm{C}$ and were subsequently blocked with PBS containing $1 \%$ bovine serum albumin and $0.3 \%$ Triton X-100 (Sigma-Aldrich, St. Louis, MO, USA) for $1 \mathrm{~h}$ at $37^{\circ} \mathrm{C}$. Sections were subsequently incubated with a primary monoclonal rat anti-BrdU antibody (1:500; catalog no., OBT 0030; AbD Serotec, Raleigh, NC, USA) overnight at $4{ }^{\circ} \mathrm{C}$, followed by incubation with the secondary antibody (Alexa Fluor 594-conjugated polyclonal donkey anti-rat; 1:400; Invitrogen; Thermo Fisher Scientific, Waltham, MA, USA; catalog no., A-21209). BrdU ${ }^{+}$cells in the unilateral dentate gyrus (DG) of each animal were counted using a Stereo Investigator stereology system (MBF Bioscience, Williston, VT, USA). The actual section thickness was measured, and the top and bottom guard zones were defined to avoid oversampling. Measurements were finished in an equidistant series of six coronal sections spanning the DG in its rostrocaudal extension. Representative confocal micrographs were obtained with a Zeiss LSM 710 confocal laser-scanning microscope (Carl Zeiss AG, Oberkochen, Germany).

ELISA. Serum was separated from trunk blood $24 \mathrm{~h}$ following the LPS injection by centrifugation at 4,000 $\mathrm{x} \mathrm{g}$ for $5 \mathrm{~min}$ and was subsequently stored at $-20^{\circ} \mathrm{C}$ until use. Serum corticosterone levels were measured using ELISA kits (Corticosterone ELISA kit; EIAab Science Co, Ltd., Wuhan, China), according to the manufacturer's instructions. The concentrations of interleukin (IL)-1 $\beta$, interferon (IFN)- $\gamma$, tumor necrosis factor (TNF)- $\alpha$, IL-6, IL-4, IL-10, brain-derived neurotrophic factor (BDNF) and insulin-like growth factor (IGF)-1 in several brain zones were determined using commercially available ELISA assays, according to the instructions supplied by the manufacturer (Mouse IL-1 $\beta$ ELISA set, Mouse IFN- $\gamma$ (AN-18) ELISA set, Mouse IL-4 ELISA set, Mouse IL-10 ELISA set; all purchased from BD Pharmingen ${ }^{\mathrm{TM}}$; BD Biosciences, Franklin Lakes, NJ, USA Mouse TNF- $\alpha$ ELISA kit, Mouse IL-6 ELISA kit, Mouse BDNF ELISA kit, Mouse IGF-1 ELISA kit; all purchased from EIAab Science Co, Ltd.).

High-performance liquid chromatography analyses of 5-HT and 5-hydroxyindoleacetic acid (5-HIAA). Brain samples were weighed and subsequently homogenized in $0.5 \mathrm{ml}$ ice-cold solution of $0.1 \mathrm{M}$ perchloric acid, containing $0.1 \%$ cysteine, and were centrifuged at $20,817 \mathrm{x}$ g for $20 \mathrm{~min}$ at $4^{\circ} \mathrm{C}$. The standard solution or sample was then injected onto the column $\left(5 \mu \mathrm{m} ; 4.6 \times 150 \mathrm{~mm}^{2}\right)$. The separation was performed on a reversed-phase Hypersil BDS-C18 column (Elite Analytical Instruments Co., Ltd., Dalian, China) in an isocratic elution mode using a mobile phase consisting of $85 \mathrm{mM}$ citric acid and $100 \mathrm{mM}$ sodium acetate buffer ( $\mathrm{pH} 4.0)$, containing $8 \%$ methanol, $3 \mathrm{mM}$ sodium heptane-1-sulphonate and $0.2 \mathrm{mM}$
EDTA, at a flow rate of $0.8 \mathrm{ml} / \mathrm{min}$. The levels of 5-HT and 5-HIAA were expressed in ng/mg tissue weight (wet).

Statistical analyses. All the data were processed using SPSS version 17.0 for Windows (SPSS, Inc., Chicago, IL, USA). The data are expressed as mean \pm standard error. Data from the SEB, FST and TST were analyzed using three-way (BCGxLPSxtime) repeated measures analysis of variance (ANOVA) followed by Bonferroni post-hoc test. Data from the remaining tests were analyzed using two-way (BCGxLPS) ANOVA followed by Bonferroni post-hoc test. $\mathrm{P}<0.05$ was considered to indicate a statistically significant difference.

\section{Results}

Neonatal BCG vaccination alleviates LPS-induced sickness behavior in adulthood. SEB was measured 30 min prior to, and 4, 8 and $24 \mathrm{~h}$ following, LPS injection. A three-way ANOVA revealed the significant effects of $\mathrm{BCG}\left(\mathrm{F}_{1,60}=12.10 ; \mathrm{P}<0.001\right)$, LPS $\left(\mathrm{F}_{1,60}=141.58 ; \mathrm{P}<0.001\right)$ and time $\left(\mathrm{F}_{3,180}=52.87 ; \mathrm{P}<0.001\right)$, and the significant interactions of BCGxLPS $\left(\mathrm{F}_{1,60}=15.13\right.$; $\mathrm{P}<0.001)$, BCGxtime $\left(\mathrm{F}_{3,180}=2.74 ; \mathrm{P}=0.0448\right)$, LPSxtime $\left(\mathrm{F}_{3,180}=49.12 ; \mathrm{P}<0.001\right)$ and BCGxLPSxtime $\left(\mathrm{F}_{3,180}=4.26\right.$; $\mathrm{P}=0.0062)$. Subsequent analyses revealed that adult LPS treatment decreased the social behavior of mice (LPS vs. CON group, $\mathrm{P}<0.001)$ and that neonatal $\mathrm{BCG}$ vaccination significantly attenuated LPS-induced sickness behavior (BCG/LPS vs. LPS group, $\mathrm{P}<0.001$; Fig. 1A).

Food intake within the $24 \mathrm{~h}$ subsequent to LPS injection was analyzed using two-way (BCGxLPS) ANOVA. There were significant effects of LPS $\left(\mathrm{F}_{1,36}=62.35 ; \mathrm{P}<0.001\right)$ and interaction of BCGxLPS $\left(\mathrm{F}_{1,36}=7.47 ; \mathrm{P}=0.0097\right)$. Subsequent analyses revealed that adult LPS treatment decreased the food intake (LPS vs. CON group, $\mathrm{P}<0.001$ ) and that neonatal BCG vaccination significantly attenuated the LPS-induced decrease (BCG/LPS vs. LPS group, $\mathrm{P}=0.0019$; Fig. 1B).

A two-way ANOVA of body weight change within the initial $24 \mathrm{~h}$ following LPS injection revealed a significant effect of BCG $\left(\mathrm{F}_{1,36}=561.57 ; \mathrm{P}<0.001\right)$, LPS $\left(\mathrm{F}_{1,36}=95.25 ; \mathrm{P}<0.001\right)$ and interaction of BCGxLPS $\left(\mathrm{F}_{1,36}=91.38 ; \mathrm{P}<0.001\right)$. Subsequent analyses revealed that adult LPS treatment decreased the body weight of the mice (LPS vs. CON group, $\mathrm{P}<0.001$ ) and that neonatal $\mathrm{BCG}$ vaccination significantly attenuated the LPS-induced decrease (BCG/LPS vs. LPS group, $\mathrm{P}<0.001$; Fig. 1C).

Notably, neonatal BCG vaccination alone had a non-significant impact on social behavior, food intake and body weight in adulthood compared with the $\mathrm{CON}$ group (P-values for comparisons between the $\mathrm{BCG}$ and $\mathrm{CON}$ groups for social behavior: $\mathrm{P}=0.3212$; food intake: $\mathrm{P}=0.6041$; and body weight: $\mathrm{P}=0.8881$; Fig. 1).

Neonatal BCG vaccination weakens LPS-induced depression and anxiety-like behaviors in adulthood. The mice were subjected to the FST and TST tasks $30 \mathrm{~min}$ prior to, and 4,8 and $24 \mathrm{~h}$ following, LPS injection. A three-way (BCGxLPSxtime) ANOVA of the FST data revealed significant effects of BCG $\left(\mathrm{F}_{1,60}=31.42 ; \mathrm{P}<0.001\right)$, LPS $\left(\mathrm{F}_{1,60}=125.44\right.$; $\mathrm{P}<0.001)$ and time $\left(\mathrm{F}_{3,180}=36.13 ; \mathrm{P}<0.001\right)$, and significant interactions of BCGxLPS $\left(\mathrm{F}_{1,60}=28.26\right.$; $\left.\mathrm{P}<0.001\right)$, BCGxtime 
$\left(\mathrm{F}_{3,180}=3.01 ; \mathrm{P}=0.0316\right)$, LPSxtime $\left(\mathrm{F}_{3,180}=36.26 ; \mathrm{P}<0.001\right)$ and BCGxLPSxtime $\left(\mathrm{F}_{3,180}=4.86 ; \mathrm{P}=0.0028\right)$. Subsequent analyses revealed that adult LPS treatment increased the immobility time of mice (LPS vs. CON group, $\mathrm{P}<0.001$ ) and that neonatal BCG vaccination significantly attenuated the LPS-induced increase (BCG/LPS vs. LPS group, $\mathrm{P}<0.001$; Fig. $2 \mathrm{~A}$ ).

Analyses of the TST data revealed significant effects of BCG $\left(\mathrm{F}_{1,60}=11.83, \mathrm{P}=0.0011\right)$, LPS $\left(\mathrm{F}_{1,60}=81.77, \mathrm{P}<0.001\right)$ and time $\left(\mathrm{F}_{3,180}=15.07, \mathrm{P}<0.001\right)$, and significant interactions of BCGxLPS $\left(\mathrm{F}_{1,60}=16.17, \mathrm{P}<0.001\right)$, BCGxtime $\left(\mathrm{F}_{3,180}=3.77, \mathrm{P}=0.0117\right)$, LPSxtime $\left(\mathrm{F}_{3,180}=16.32, \mathrm{P}<0.001\right)$ and BCGxLPSxtime $\left(\mathrm{F}_{3,180}=5.56, \mathrm{P}=0.0011\right)$. Subsequent analyses revealed that adult LPS treatment increased the immobility time of mice (LPS vs. CON group, $\mathrm{P}<0.001$ ) and that neonatal $B C G$ vaccination attenuated the LPS-induced increase (BCG/LPS vs. LPS group, P<0.001; Fig. 2B).

The mice were subjected to the OFT task $4 \mathrm{~h}$ subsequent to the LPS injection, and locomotor activities, rearing activities and the proportion of center area activities in total were recorded for $30 \mathrm{~min}$. A two-way ANOVA revealed significant effects of BCG (rearing activities, $\mathrm{F}_{1,60}=7.01 ; \mathrm{P}=0.0103$; center proportion, $\mathrm{F}_{1.60}=4.01 ; \mathrm{P}=0.0497$ ) and LPS (locomotor activities, $\mathrm{F}_{160}=7.86 ; \mathrm{P}=0.0067$; rearing activities, $\mathrm{F}_{1.60}=31.58$; $\mathrm{P}<0.001$; center proportion, $\left.\mathrm{F}_{1,60}=30.61 ; \mathrm{P}<0.001\right)$ and interaction of BCGxLPS (center proportion, $\mathrm{F}_{1,60}=4.25 ; \mathrm{P}=0.0435$ ). Subsequent analyses revealed that LPS injection alone induced a marginal, but significant, decrease in locomotor activities (LPS vs. CON group, $\mathrm{P}<0.01$ ) and that neonatal $\mathrm{BCG}$ vaccination did not significantly affect the locomotor activities, regardless of adult LPS treatment (BCG vs. CON group, $\mathrm{P}=0.601 ; \mathrm{BCG} / \mathrm{LPS}$ vs. LPS group, $\mathrm{P}=0.092)$. Subsequent analyses for the other indices of OFT revealed that adult LPS treatment induced decreases in locomotion, rearing activities (LPS vs. CON group, $\mathrm{P}<0.001$ ) and center proportion activities (LPS vs. CON group, $\mathrm{P}<0.001$ ). Neonatal $\mathrm{BCG}$ vaccination attenuated the LPS-induced decreases in rearing activities (BCG/LPS vs. LPS group, $\mathrm{P}<0.001$ ) and center proportion (BCG/LPS vs. LPS group, $\mathrm{P}<0.001$; Fig. 2C-E).

Notably, neonatal BCG vaccination alone insignificantly impacted these indices in the FST and TST tasks in adulthood compared with the CON group (Fig. 2).

Neonatal BCG vaccination alleviates the LPS-induced impairment in hippocampal cell proliferation. There were significant effects of $\mathrm{BCG}\left(\mathrm{F}_{1,20}=6.761 ; \mathrm{P}=0.0171\right)$ and, LPS $\left(\mathrm{F}_{1,20}=70.485 ; \mathrm{P}<0.001\right)$ and interaction of BCGxLPS $\left(F_{1,20}=6.645 ; P=0.0180\right)$. Subsequent analyses revealed that adult LPS treatment decreased the number of $\mathrm{BrdU}^{+}$cells (LPS vs. $\mathrm{CON}$ group, $\mathrm{P}<0.001)$ and that neonatal $\mathrm{BCG}$ vaccination attenuated LPS-induced impairment (BCG/LPS vs. LPS group, $\mathrm{P}=0.0016$ ), although neonatal $\mathrm{BCG}$ vaccination alone caused no significant effect (BCG vs. $\mathrm{CON}$ group, $\mathrm{P}=0.9875$; Fig. 3).

Neonatal BCG vaccination reduces the LPS-induced proinflammatory cytokine response in serum and the brain. LPS treatment induced proinflammatory cytokine expression in serum and the brain. Neonatal BCG vaccination reduced the LPS-induced proinflammatory responses in the serum and brain, although neonatal BCG vaccination alone resulted in
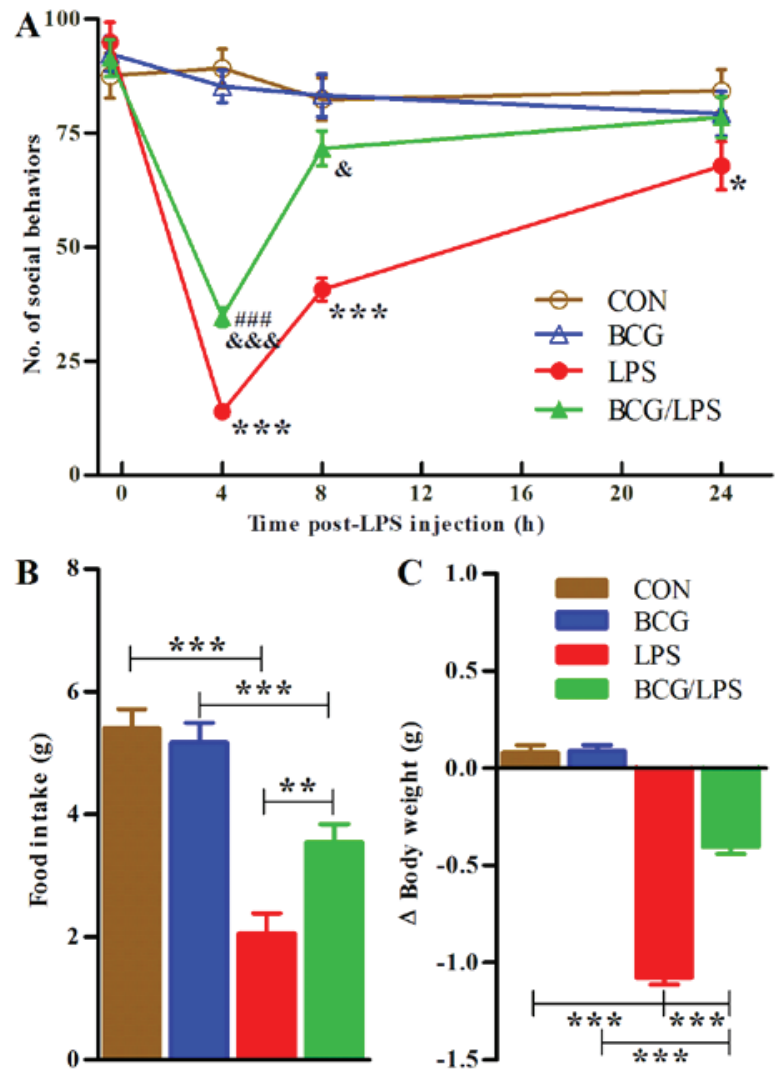

Figure 1. Neonatal BCG vaccination alleviates LPS-induced sickness behavior in adulthood. (A) Social exploratory behavior was measured $30 \mathrm{~min}$ prior to the LPS injection and 4, 8 , and $24 \mathrm{~h}$ later. The data were analyzed using three-way (BCGxLPSxtime) repeated measures ANOVA followed by Bonferroni post-hoc test. The data represent the mean \pm SE $(n=16)$. In a different experiment, (B) food intake and (C) body weight changes were measured within $24 \mathrm{~h}$ of the LPS injection. The symbol ' $\Delta$ ' in ' $\Delta$ Body weight' means alteration in body weight. The data were analyzed using two-way (BCGxLPS) ANOVA followed by Bonferroni post-hoc test. The bars represent the mean $\pm \mathrm{SE}(\mathrm{n}=10)$. The symbols '*', '\#' and ' $\&$ ' indicate significant differences compared with the CON mice, BCG group and LPS group, respectively. The single, double and triple symbols indicate $\mathrm{P}<0.05$ $\mathrm{P}<0.01$ and $\mathrm{P}<0.001$, respectively (post-hoc differences). The experiment was repeated twice with similar results. BCG, Bacillus Calmette-Guérin; LPS, lipopolysaccharide; ANOVA, analysis of variance; SE, standard error; CON, control.

no significant alteration in cytokine expression in serum or the brain (Fig. 4). The statistical analyses of two-way ANOVA for cytokines are presented in Table I.

In the serum, the LPS group exhibited significantly increased levels of IL-1 $\beta$, IFN- $\gamma$, TNF- $\alpha$ and IL- 6 compared with the CON group; the BCG/LPS group exhibited significantly reduced levels of IL-1 $\beta$, IFN- $\gamma$ and IL- 6 and increased levels of IL-10 compared with the LPS group (Fig. 4A). In the hippocampus, the LPS group exhibited significantly increased levels of IL-1 $\beta$ and IL- 6 compared with the CON group; the BCG/LPS group exhibited significantly reduced levels of IL-1 $\beta$ and IL- 6 and increased levels of IL-10 compared with the LPS group (Fig. 4B). In the prefrontal cortex, the LPS group exhibited significantly increased levels of IL-1 $\beta$, TNF- $\alpha$, IL-6 and IL-10 and reduced levels of IL-4 compared with the CON group; the BCG/LPS group exhibited significantly reduced levels of IL-1 $\beta$, TNF- $\alpha$ and IL- 6 and increased levels of IL-4 and IL-10 compared with the LPS group (Fig. 4C). In 


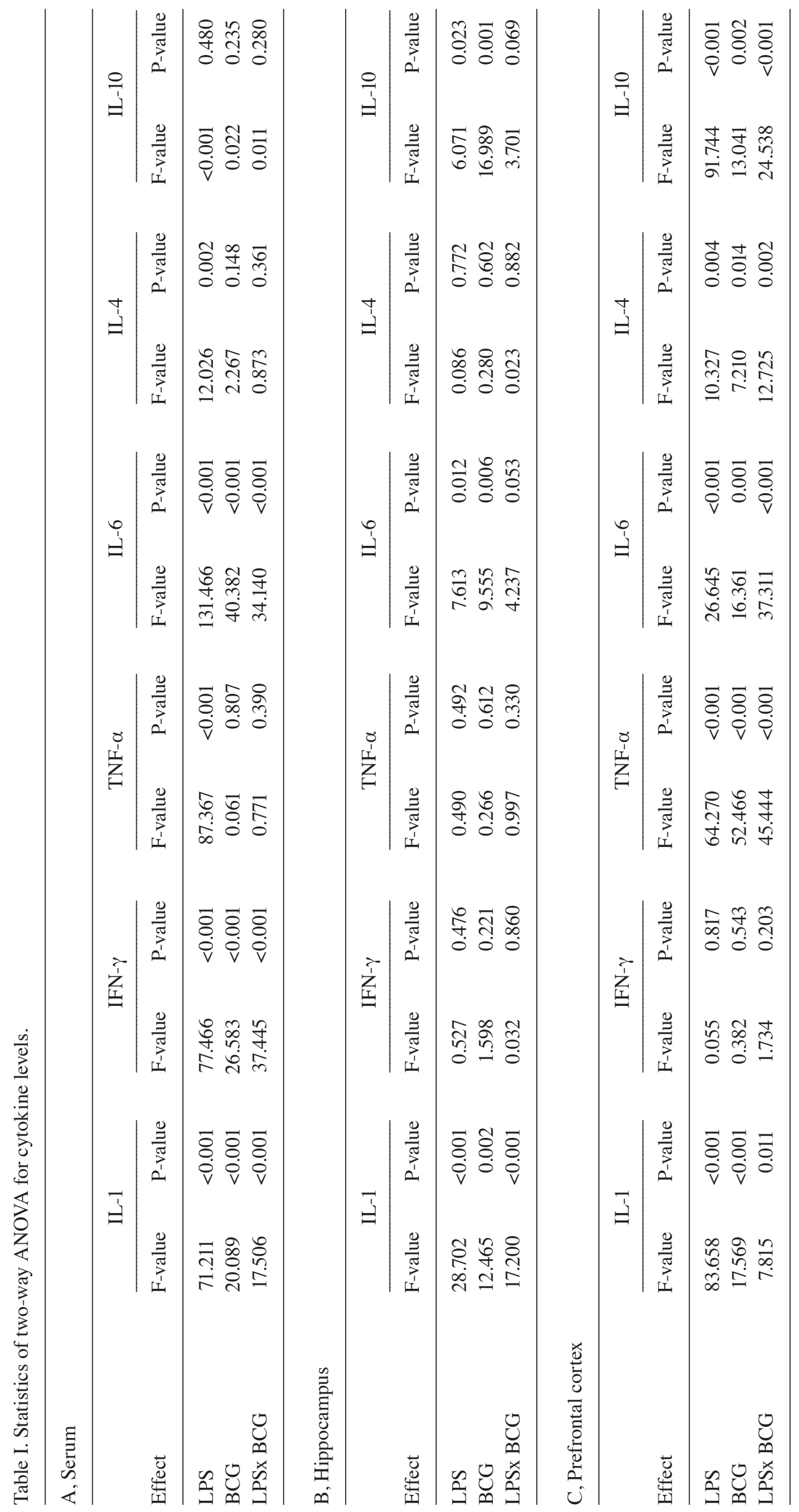


the striatum, the LPS group exhibited significantly increased levels of IL-1 $\beta$, TNF- $\alpha$, IL-6 and IL-10 compared with the CON group; the BCG/LPS group exhibited significantly reduced levels of IL-1 $\beta$, TNF- $\alpha$, and IL- 6 and increased levels of IL-10 compared with the LPS group (Fig. 4D).

In addition, the levels of IFN- $\gamma$ in serum, the levels of IL-1 $\beta$ and IL- 6 in the hippocampus, the levels of TNF- $\alpha$, IL- 6 in the prefrontal cortex and the levels of TNF- $\alpha$ in the striatum of the $\mathrm{BCG} / \mathrm{LPS}$ group were as low as their levels in the BCG group (Fig. 4). Therefore, neonatal BCG vaccination completely prevented LPS-induced increased expression of certain proinflammatory cytokines in the serum or specific zones of the brain. In addition, compared with the BCG group, the levels of the anti-inflammatory cytokines, IL-4 and IL-10, in serum and the brain were elevated in the BCG/LPS group, although adult LPS treatment alone had a marginal and inconsistent influence on the levels of IL-4 and IL-10 (Fig. 4).

Neonatal BCG vaccination weakens the LPS-induced decrease in BDNF and IGF-1 levels in the brain. Adult LPS treatment reduced levels of the neurotrophins BDNF and IGF-1 in some or all of the three brain zones investigated. Neonatal BCG vaccination weakened these LPS-induced decreases, although neonatal BCG vaccination alone did not significantly alter the levels of these neurotrophins (Fig. 5). The statistical analysis of two-way ANOVA are presented in Table II.

The LPS group exhibited significantly reduced BDNF levels in the hippocampus, prefrontal cortex and striatum compared with the CON group; the BCG/LPS group exhibited significantly increased BDNF levels in all three zones compared with the LPS group (Fig. 5). The LPS group exhibited significantly reduced IGF-1 levels in prefrontal cortex and striatum compared with the CON group; the BCG/LPS group exhibited significantly increased IGF-1 levels in the prefrontal cortex and striatum compared with the LPS group (Fig. 5). In addition, the levels of BDNF in the hippocampus and IGF-1 in the striatum of the BCG/LPS group were as high as their levels in the BCG group (Fig. 5).

Neonatal BCG vaccination reduces the LPS-induced increased 5-HT turnover and decreased 5-HT levels in the brain. Adult LPS treatment increased 5-HT turnover and decreased 5-HT levels in the brain. Neonatal BCG vaccination lessened these LPS-induced alterations, although neonatal BCG vaccination alone did not result in any significant alteration in these indices (Fig. 6A-F). The statistical analysis of two-way ANOVA are presented in Table II.

The LPS group exhibited a significantly increased ratio of 5-HIAA to 5-HT (5-HIAA/5-HT) and decreased 5-HT levels in the brain compared with the CON group. The BCG/LPS group had significantly decreased 5-HIAA/5-HT and increased 5-HT levels in the brain compared with the LPS group (Fig. 6A-F).

Neonatal BCG vaccination did not affect the LPS-induced increase in corticosterone levels in serum or the brain. A two-way ANOVA for corticosterone levels revealed a significant effect of LPS $\left(\mathrm{F}_{1,20}=120.942 ; \mathrm{P}<0.001\right)$, non-significant effect of BCG $\left(\mathrm{F}_{1,20}=0.842 ; \mathrm{P}=0.3697\right)$ and non-significant interaction of BCGxLPS $\left(\mathrm{F}_{1,20}=0.092 ; \mathrm{P}=0.7650\right)$. Subsequent 

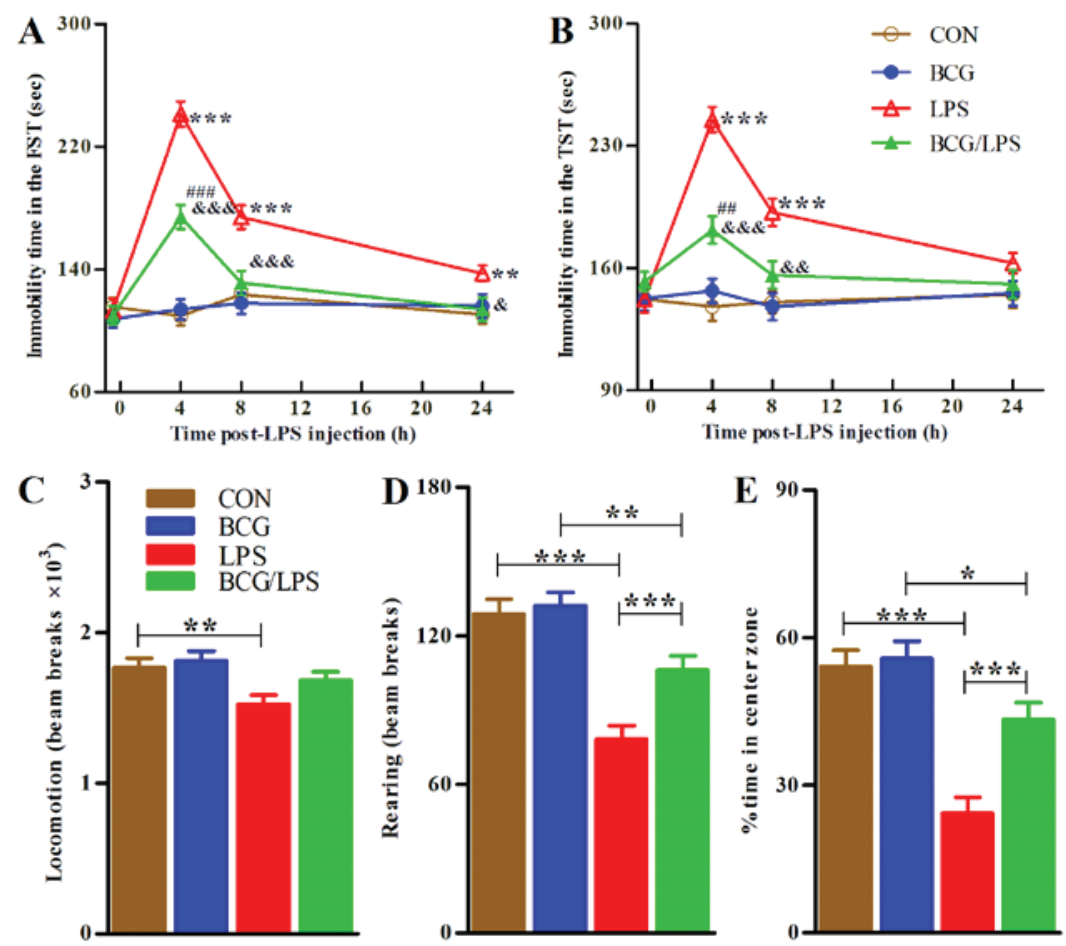

Figure 2. Neonatal BCG vaccination alleviates LPS-induced depression and anxiety-like behavior in adulthood. The mice were subjected to the FST and TST tasks 30 min prior to LPS injection and 4, 8, and $24 \mathrm{~h}$ later. The bars represent the mean immobility time spent in the (A) FST and (B) TST tasks. The data were analyzed using three-way (BCGxLPSxtime) repeated measures ANOVA followed by Bonferroni post-hoc test. The mice were subjected to the OFT task $2.5 \mathrm{~h}$ subsequent to LPS injection. The bars represent the mean (C) locomotor activities, (D) rearing activities and the (E) proportion of center zone activities by mice during the 30-min test. For each of the three behavior tests, a new set of animals were used to avoid possible disturbances. The data represent the mean \pm SE (n=16) and were analyzed using two-way ANOVA followed by Bonferroni post-hoc test. The symbols ',", '\#' and '\&' indicate significant differences compared with the CON mice, BCG group and LPS group, respectively. The single, double and triple symbols indicate $\mathrm{P}<0.05, \mathrm{P}<0.01$ and $\mathrm{P}<0.001$, respectively (post-hoc differences). The experiment was repeated twice with similar results. BCG, Bacillus Calmette-Guérin; LPS, lipopolysaccharide; FST, forced swimming test; TST, tail suspension test; ANOVA, analysis of variance; OFT, open field test; SE, standard error; CON, control.

analyses revealed that the BCG group exhibited no significant alteration in the corticosterone levels in the serum compared with the CON group (Fig. 6G). The LPS group had significantly increased corticosterone levels in the serum compared with the CON group, and no significant differences were observed in the corticosterone levels in the serum between the BCG/LPS and LPS groups (Fig. 6G).

\section{Discussion}

The results of the present study reveal that neonatal BCG vaccination alleviates LPS-induced neurobehavioral impairments and neuroinflammation in adult mice. Neonatal BCG vaccination alleviated LPS-induced sickness, depression and anxiety-like behaviors, as well as hippocampal proliferation impairment. Furthermore, LPS-induced decreases in neurotrophins and 5-HT levels in brain were also alleviated by neonatal $B C G$ vaccination. In addition, neonatal BCG vaccination reduced the pro-inflammatory responses induced by adult LPS challenge in the periphery and brain.

Intraperitoneal LPS treatment has been confirmed to induce a series of acute physiopathological and psychological disorders in rodents. Previous studies have demonstrated decreased social exploratory behavior and food intake in rodents intraperitoneally administered with LPS $(15,23)$, while others have reported LPS-induced depression and anxiety-like behaviors $(24,25)$. Furthermore, LPS may acutely inhibit the proliferation of stem cells in the DG in adult rodents $(26,27)$. The results from the LPS group in the present study confirmed these widely reported neurobehavioral impairments.

Immune activation by LPS may induce a large release of cytokines in the periphery and brain, particularly proinflammatory cytokines, including IL- $1 \beta$, IL- 6 , TNF- $\alpha$ and IFN- $\gamma(15)$. These proinflammatory cytokines may affect the functioning of the brain (28) and mediate sickness behavior syndrome (29). For example, Wang et al (22) reported that an intraperitoneal injection of LPS in mice resulted in clear impairments in performance of the OFT task, associated with increased expression of IL-1 $\beta$, IL- 6 and TNF- $\alpha$ in the brain. IL-10 and IL-4 are considered anti-inflammatory cytokines and have neuroprotective effects $(30,31)$. In the present study, LPS treatment induced proinflammatory responses in the serum and brain. The increases in proinflammatory cytokine levels by adult LPS treatment were reduced significantly by neonatal BCG vaccination, and in certain cases cytokine levels in the serum or specific brain zones were completely prevented from increasing. These findings reveal that neonatal priming of the immune system by BCG results in a reduced proinflammatory response to a subsequent LPS challenge in adulthood, possibly explaining why neonatal BCG vaccination alleviates LPS-induced behavior impairments.

Notably, in the present study the levels of IL-10 in the LPS group demonstrated a tendency to increase in serum and all three brain zones compared with the CON group. IL-10 is 
one of the regulatory $\mathrm{T}$ cell (Tregs) associated cytokines that may be released by various immune cells when inflammation occurs (32). Stumhofer et al (33) reported that IL-6 induced signal transducers and activators of transcription 3-mediated T cell production of IL-10. McGeachy et al (34) reported that IL- 6 drives the production of IL-10 by T cells and prevents the $\mathrm{T}$ helper 17 cell-mediated pathology. The increased IL-10 levels may inhibit inflammatory pathologies and avoid autoimmune impairments (35).

Notably, in the present study the BCG/LPS group exhibited increased IL-10 levels in the periphery and brain compared with the BCG and LPS groups. This observation aids explanation of the reduced levels of proinflammatory cytokines in the BCG/LPS group. How neonatal BCG vaccination increases IL-10 production in response to adult LPS treatment remains to be elucidated. However, studies concerning the non-specific effects of BCG on the immune system support the anti-inflammatory role of BCG (36-38). Epidemiological studies have indicated that BCG vaccination exerted a positive non-specific effect on overall childhood mortality, which cannot be attributed to the prevention of tuberculosis fatalities $(39,40)$. Additionally, the capacity of BCG to induce Tregs in vivo has been widely reported (36). In a study by Madura Larsen et al (37), dendritic cells (DCs) were generated from peripheral blood mononuclear cells and cultured with LPS or LPS/BCG in vitro. The study reported that BCG-exposed DCs were able to induce IL-10-producing T cells. In a separate study, neonatal BCG vaccination was observed to ameliorate allergen-induced local inflammation and increase the number of cluster of differentiation $4(\mathrm{CD} 4)^{+} \mathrm{CD} 25^{+}$Treg cells and IL-10 expression (38). These findings suggest that BCG may interact with DCs directly to result in an accumulation of IL-10-producing T cells.

DCs derived from BCG-infected mononuclear cells produce IL-4, which may be associated with the failure of the BCG vaccination against tuberculosis (41). This suggests that IL-4-producing DCs may have an anti-inflammatory role. In the present study, increased expression of IL-4 was observed in serum and the prefrontal cortex of the BCG/LPS compared with the LPS mice, suggesting that increased IL-4 may contribute to the inhibition of LPS-induced inflammation.

BDNF, IGF-1 and 5-HT in the brain were identified as the most important molecules for maintaining health-mood status, and decreases in their levels, as well as an increase in 5-HT turnover, are associated with depression $(42,43)$. In the present study, behavior impairments and alterations in neurotrophins and 5-HT levels in LPS mice were consistent with these reports. Furthermore, neonatal BCG vaccination improved the LPS-induced neurochemical disorders. This alleviation, together with the changes in proinflammatory cytokine levels, may assist with explaining the effects of BCG on behavior and proliferation observed in the present study.

Activity of the hypothalamic-pituitary-adrenal (HPA) axis is another important aspect of the complicated pathophysiology of sickness-like behavior and depression. HPA axis hyperactivity may be one of the mechanisms underlying depression development (44). Serum levels of corticosterone are one marker of the HPA axis activation in rodents (45). As reported in our previous study, LPS treatment increased serum corticosterone levels in mice (46). However, in the

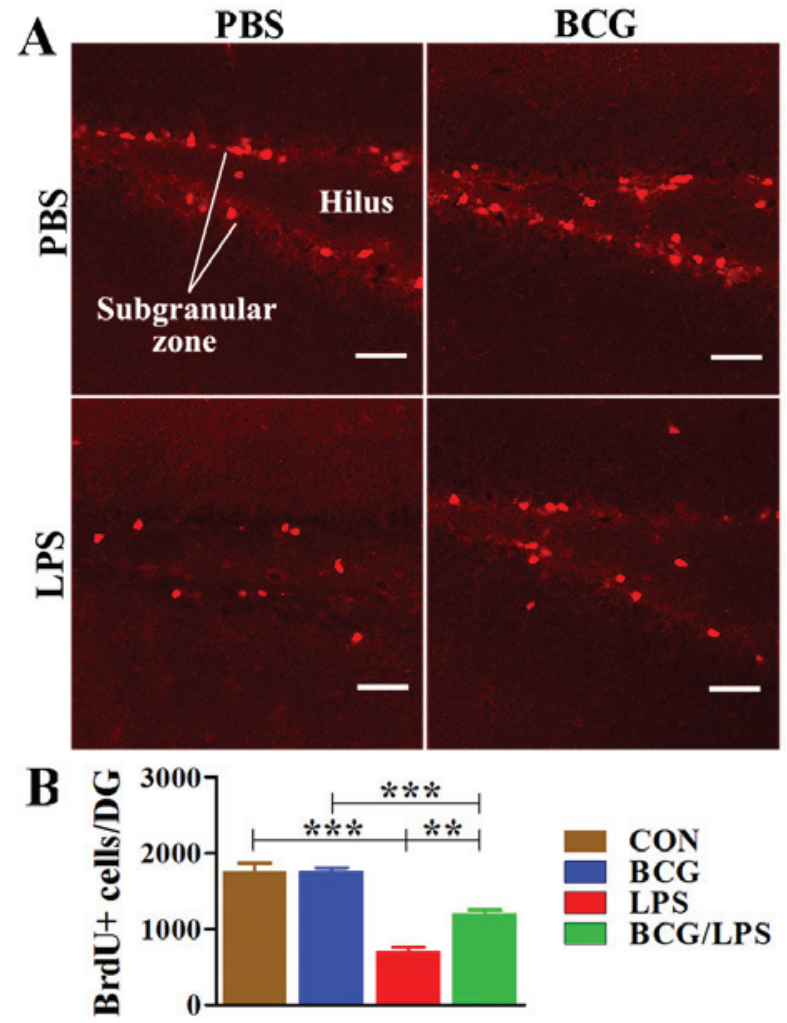

Figure 3. Neonatal BCG vaccination alleviates LPS-induced impairment in hippocampal cell proliferation. (A) Representative confocal micrographs of DG in the CON (upper left panel), BCG (upper right panel), LPS (lower left panel) and BCG/LPS groups (lower right panel). (B) The bars represent the mean $\pm \mathrm{SE}$ of the cell number in each group. The data represent the mean $\pm S E(n=6)$ and were analyzed using two-way analysis of variance followed by Bonferroni post-hoc test. ${ }^{* *}$ and ${ }^{* * *}$ indicate $\mathrm{P}<0.01$ and $\mathrm{P}<0.001$, respectively (post-hoc differences). Scale bar $=50 \mu \mathrm{m}$. The experiment was repeated twice with similar results. BCG, Bacillus Calmette-Guérin; LPS, lipopolysaccharide; DG, dentate gyrus; CON, control; SE, standard error; PBS, phosphate-buffered saline; BrdU, 5-bromo-2-deoxyuridine.

present study neonatal BCG vaccination did not influence the LPS-induced elevation of serum corticosterone levels, suggesting that neonatal BCG vaccination lessened the LPS-induced neurobehavioral impairments through its priming effects on inflammatory responses in the periphery and brain, independent of the HPA axis.

How peripheral cytokines exert their influence on the brain and itsfunction remains to be fully elucidated.Periphery-derived cytokines may permeate across the blood-brain barrier and directly affect the neuronal activities (47). Additionally, crosstalk may occur between cytokines in the brain and resident immune cells (such as microglia), causing the latter to change their phenotype by, for example, altering their secretion of local neuromodulative molecules, including cytokines and neurotrophins $(48,49)$. These neuromodulative molecules and local cells combine to regulate the neuroimmune niche and therefore affect brain functions $(43,50)$.

There are numerous studies reporting the influence of previous exposure to a specific antigen on subsequent immune responses to other antigen(s), including i) the effects of neonatal exposure to immunogen and subsequent autism/schizophrenia-like behavior; ii) the target disease-specific effects of a previous vaccination on the subsequent immune responses to unrelated antigens; and iii) the phenomenon of 
Table II. Statistics of two-way ANOVA for neurotrophins, 5-HT turnover and 5-HT levels.

A, Hippocampus

\begin{tabular}{|c|c|c|c|c|c|c|c|c|}
\hline \multirow[b]{2}{*}{ Effect } & \multicolumn{2}{|c|}{ BDNF } & \multicolumn{2}{|c|}{ IGF-1 } & \multicolumn{2}{|c|}{ 5-HIAA/5-HT } & \multicolumn{2}{|c|}{$5-\mathrm{HT}$} \\
\hline & F-value & P-value & F-value & P-value & F-value & P-value & F-value & P-value \\
\hline LPS & 7.751 & 0.011 & 1.545 & 0.228 & 65.638 & 0.000 & 39.781 & 0.000 \\
\hline $\mathrm{BCG}$ & 4.082 & 0.057 & 0.714 & 0.408 & 6.445 & 0.020 & 8.429 & 0.009 \\
\hline LPSx BCG & 13.601 & 0.001 & 0.175 & 0.680 & 3.460 & 0.078 & 6.003 & 0.024 \\
\hline
\end{tabular}

B, Prefrontal cortex

\begin{tabular}{|c|c|c|c|c|c|c|c|c|}
\hline \multirow[b]{2}{*}{ Effect } & \multicolumn{2}{|c|}{ BDNF } & \multicolumn{2}{|c|}{ IGF-1 } & \multicolumn{2}{|c|}{ 5-HIAA/5-HT } & \multicolumn{2}{|c|}{ 5-HT } \\
\hline & F-value & P-value & F-value & P-value & F-value & P-value & F-value & P-value \\
\hline LPS & 35.191 & 0.000 & 3.601 & 0.072 & 57.328 & 0.000 & 51.183 & 0.000 \\
\hline $\mathrm{BCG}$ & 0.426 & 0.522 & 5.057 & 0.036 & 5.315 & 0.032 & 11.952 & 0.002 \\
\hline LPSx BCG & 6.630 & 0.018 & 12.430 & 0.002 & 7.378 & 0.013 & 9.378 & 0.006 \\
\hline
\end{tabular}

C, Striatum

\begin{tabular}{|c|c|c|c|c|c|c|c|c|}
\hline \multirow[b]{2}{*}{ Effect } & \multicolumn{2}{|c|}{ BDNF } & \multicolumn{2}{|c|}{ IGF-1 } & \multicolumn{2}{|c|}{ 5-HIAA/5-HT } & \multicolumn{2}{|c|}{$5-\mathrm{HT}$} \\
\hline & F-value & P-value & F-value & P-value & F-value & P-value & F-value & P-value \\
\hline LPS & 59.356 & 0.000 & 23.287 & 0.000 & 41.972 & 0.000 & 35.541 & 0.000 \\
\hline BCG & 3.497 & 0.076 & 3.055 & 0.096 & 4.963 & 0.038 & 2.025 & 0.170 \\
\hline LPSx BCG & 9.024 & 0.007 & 1.595 & 0.221 & 7.906 & 0.011 & 5.044 & 0.036 \\
\hline
\end{tabular}

Data in this table represent the F- and P-values from two-way ANOVA for cytokine levels in the hippocampus, prefrontal cortex and striatum. LPS, main effect of LPS $\left(\mathrm{F}_{1,20}\right)$; BCG, main effect of BCG $\left(\mathrm{F}_{1,20}\right)$; LPSxBCG, interaction effect of BCGxLPS $\left(\mathrm{F}_{1,20}\right)$. P $<0.05$ was considered to indicate a significant difference. ANOVA, analysis of variance; BCG, Bacillus Calmette-Guérin; LPS, lipopolysaccharide; 5-HT, 5-hydroxytryptamine; 5-HIAA, 5-hydroxyindoleacetic acid; BDNF, brain-derived neurotrophic factor; IGF-1, insulin-like growth factor-1.

'original antigenic sin'. Previous studies have demonstrated that early life immune activation by Escherichia coli led to CNS alterations at behavioral, cellular and molecular levels following adult LPS challenge $(10,14,17)$. It has additionally been observed that these later life consequences were mediated primarily by CNS resident immune cells, including microglia and astrocytes that were primed and thus equipped with altered abilities when responding to subsequent immune stimuli $(10,14,17)$. A previous study revealed that BCG vaccination enhanced the immunogenicity of subsequent influenza vaccination in healthy volunteers through enhanced proinflammatory leukocyte responses (51). Original antigenic sin describes the failure to mount effective antibody responses to virus variants in a previously virus-infected host (52). The similarity of the prior and subsequent antigens and the hyperresponsiveness of memory immune cells are thought to be the underlying reasons for this phenomenon (52). Similar mechanisms to those identified by previous studies may apply in the present study, as BCG-priming altered the expression of cytokines and neurotrophins in the brain, which are produced and/or regulated by microglia and astrocytes $(10,14,17)$. The mechanism concerning mainly peripheral cytokine responses may also underlie the findings of the present study, as indicated by the altered serum cytokines levels (51). However, the present study observed abrogation, rather than enhancement, of immunogenicity. This is understandable as BCG induces a mild, physiological immune activation (unlike early life pathology) and LPS exposure resulted in marked neuroinflammation (unlike later life vaccination) $(10,14,17,51)$. Original antigenic sin may not apply in the present study as it occurs during antibody responses to viral antigens (52).

The present study revealed a neuroprotective role for neonatal BCG vaccination in the presence of later-life neuroinflammation regardless of the lack of direct neurobehavioral effects in adulthood. Notably, there are certain previous studies reporting that rodents receiving BCG in adulthood develop a depression-like phenotype (53-55). In addition, previous studies reporting BCG depression-like effects identified that BCG activated indoleamine 2,3-dioxygenase, which is a tryptophan-catabolizing enzyme, and decreased brain 5-HT levels (54-56). In the present study, BCG/LPS mice exhibited a lower 5-HT turnover and higher levels of 5-HT 

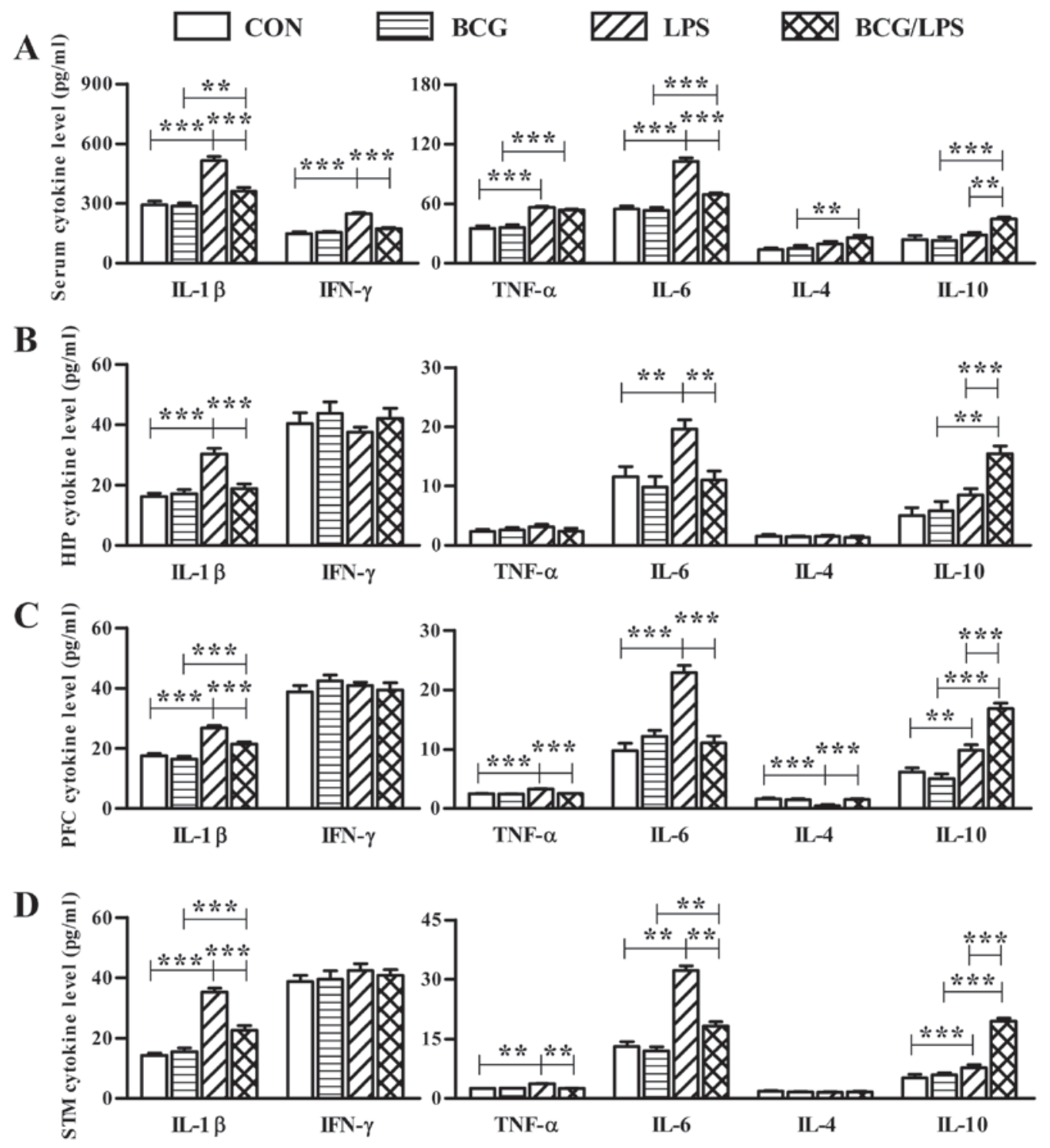

Figure 4. Neonatal BCG vaccination alleviates the LPS-induced proinflammatory cytokine response in the serum and brain. Cytokine levels (in the serum or brain tissue supernatent) were assessed $4 \mathrm{~h}$ subsequent to LPS injection. The bars represent the mean cytokine levels in (A) serum, (B) hippocampus, (C) prefrontal cortex and (D) striatum. The data represent the mean $\pm \mathrm{SE}(\mathrm{n}=6)$ and were analyzed using two-way analysis of variance followed by Bonferroni post-hoc test. ${ }^{* *}$ and ${ }^{* * *}$ indicate $\mathrm{P}<0.01$ and $\mathrm{P}<0.001$, respectively (post-hoc differences). The experiment was repeated three times with similar results. BCG, Bacillus Calmette-Guérin; LPS, lipopolysaccharide; CON, control; SE, standard error; HIP, hippocampus; PFC, prefrontal cortex; STM, striatum; IL, interleukin; IFN- $\gamma$, interferon- $\gamma$; TNF- $\alpha$, tumor necrosis factor- $\alpha$.

in the brain compared with the LPS group, suggesting that neonatal BCG vaccination may assist in maintaining normal 5-HT metabolism in adulthood. This plausible inconsistency may result from numerous factors, particularly the dosage and age for vaccination, and the time interval between BCG vaccination and behavioral tests. In the previous studies, doses of $\geq 10^{7} \mathrm{CFU}$ were used, as these higher doses were able to elicit clear sickness responses $(53,55)$. By contrast, a lower dose $\left(10^{5} \mathrm{CFU}\right)$ was selected in the present study to avoid inducing health impairment. BCG-induced depressive-like behavior and evident bodyweight loss have been verified as dose-dependent, and are induced only by $\geq 10^{7} \mathrm{CFU}$ (57). Additionally, sickness and depression-like behaviors were observed in the mice within 1 month of adult BCG vaccination in the previous studies (53-55). In the present study, newborn mice were vaccinated and their behaviors were tested at 12 weeks old. During longer time intervals across the significant postnatal development span, the organism may experience complex and sufficient self-adjustment to reverse the neurobehavioral effects of the neonatal vaccination, and if there are any, they are not detectable. Furthermore, other factors, including vaccination routes (intraperitoneally in previous studies and subcutaneously in the present study) may also contribute. Therefore, no contradiction exists between the current data and previous studies concerning adult models of depression by BCG.

Sirén et al (58) injected adult male Sprague-Dawley rats with BCG through the tail vein. LPS was then injected into the lateral cerebral ventricle 2 weeks later. This previous study reported that the incidence of paralysis and fatality in response to LPS was increased in BCG-primed rats. There are various methodological differences in the study by Sirén et al compared with the present study: The species (rats vs. mice in the present study), the age of vaccination (adult vs. neonates), 


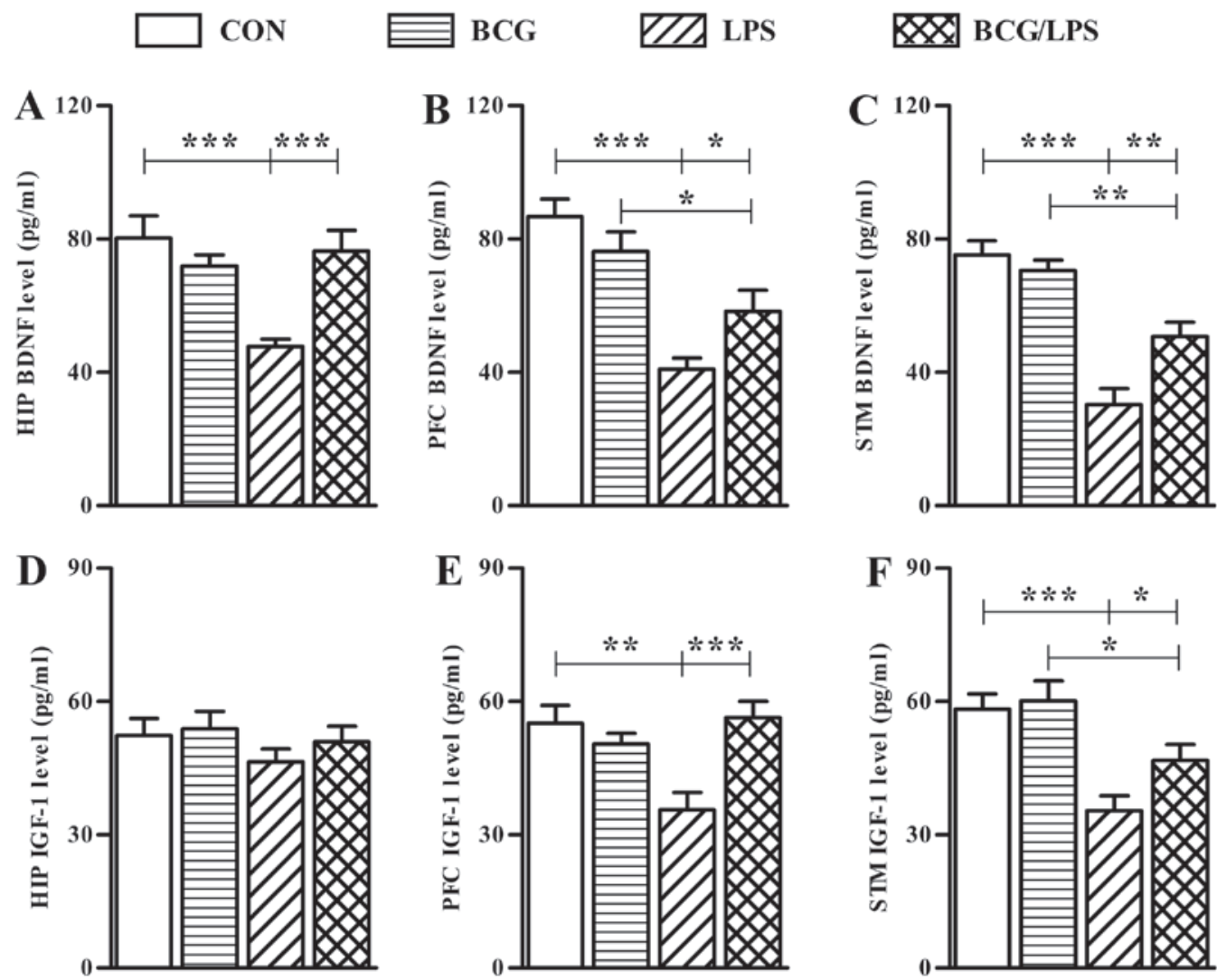

Figure 5. Neonatal BCG vaccination alleviates LPS-induced decreases in BDNF and IGF-1 levels in the brain. These molecues were examined $4 \mathrm{~h}$ subsequent to LPS injection. The bars represent the mean levels of BDNF in the (A) hippocampus, (B) prefrontal cortex and (C) striatum. The bars represent the mean levels of IGF-1 in the (D) hippocampus, (E) prefrontal cortex and (F) striatum. The data are expressed as the mean $\pm \mathrm{SE}(\mathrm{n}=6)$ and were analyzed using two-way analysis of variance followed by Bonferroni post-hoc test. ${ }^{* * *}$ and ${ }^{* * *}$ indicate $\mathrm{P}<0.05, \mathrm{P}<0.01$ and $\mathrm{P}<0.001$, respectively (post-hoc differences). The experiment was repeated three times with similar results. BCG, Bacillus Calmette-Guérin; LPS, lipopolysaccharide; CON, control; BDNF, brain-derived neurotrophic factor; IGF-1, insulin-like growth factor-1; SE, standard error; HIP, hippocampus; PFC, prefrontal cortex; STM, striatum.
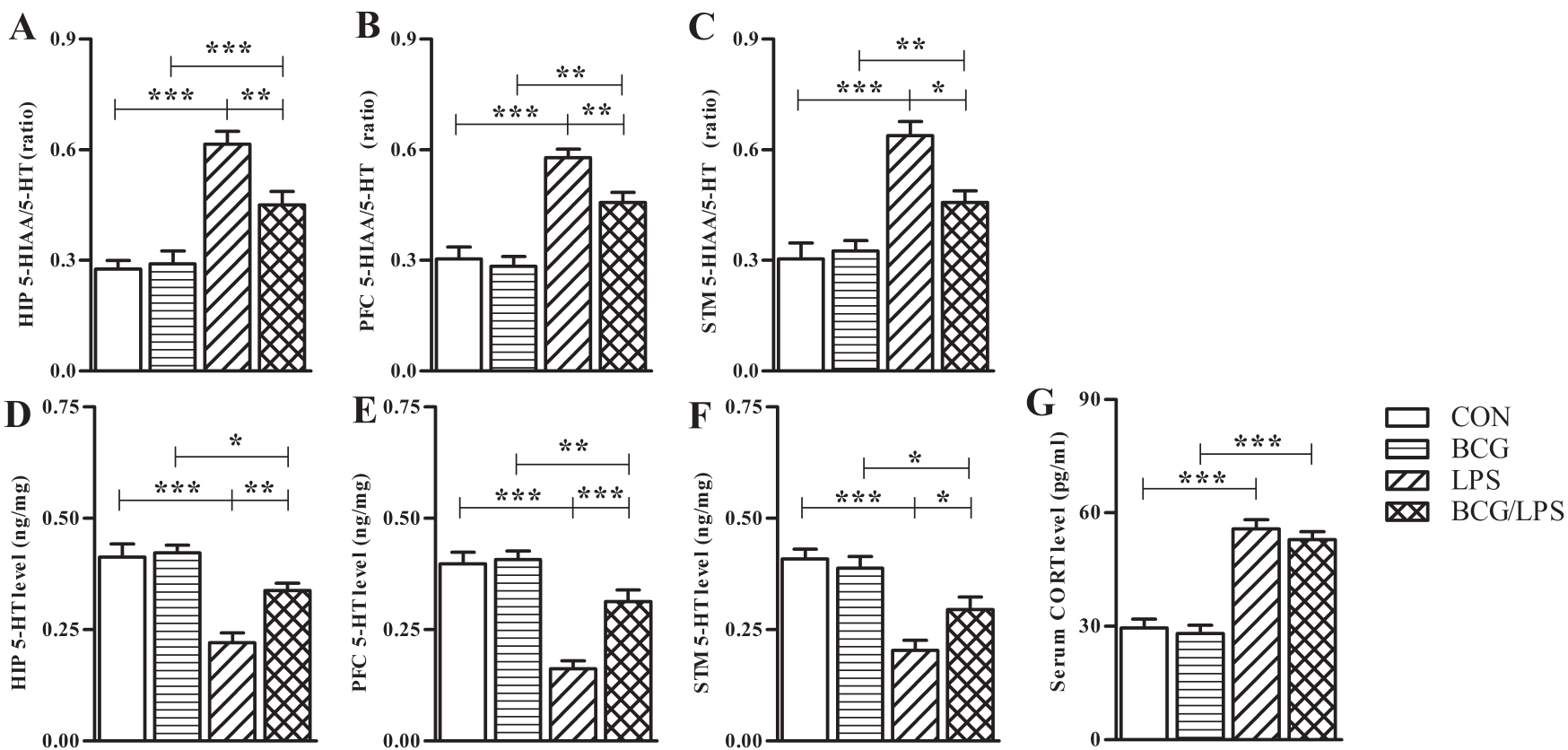

Figure 6. Neonatal BCG vaccination alleviates LPS-induced 5-HT metabolism disorder in the brain without altering the peripheral corticostrone levels. These molecues were examined $4 \mathrm{~h}$ subsequent to LPS injection. The bars represent the mean ratio of 5-HIAA to 5-HT in the (A) hippocampus, (B) prefrontal cortex and (C) striatum. The bars represent the mean levels of 5-HT in the (D) hippocampus, (E) prefrontal cortex and (F) striatum. (G) The bars represent the mean levels of corticosterone in the serum. The data are expressed as the mean $\pm \mathrm{SE}(\mathrm{n}=6)$ and were analyzed using two-way analysis of variance followed by Bonferroni post-hoc test. ${ }^{* * *}$ and ${ }^{* * *}$ indicate $\mathrm{P}<0.05, \mathrm{P}<0.01$ and $\mathrm{P}<0.001$, respectively (post-hoc differences). The experiment was repeated three times with similar results. BCG, Bacillus Calmette-Guérin; LPS, lipopolysaccharide; CON, control; 5-HT, 5-hydroxytryptamine; 5-HIAA, 5-hydroxyindoleacetic acid; CORT, corticosterone; SE, standard error; HIP, hippocampus; PFC, prefrontal cortex; STM, striatum. 
the time interval between BCG vaccination and LPS injection ( 2 vs. 12 weeks), the route of injection of BCG (through tail vein vs. subcutaneous) and LPS (into the lateral cerebral ventricle vs. intraperitoneally). Among the differences, the injection route and time interval may be the most responsible for the neurobehavioral effects of BCG/LPS administration in animals. As Sirén et al (58), described, $13 \%$ of rats were paralyzed or succumbed following injection of $300 \mu \mathrm{g}$ LPS into the lateral cerebral ventricle. However, a similar $(\sim 1.2 \mathrm{mg} / \mathrm{kg})$ or lower $(330 \mu \mathrm{g} / \mathrm{kg}$, as used in the present study) dosage of LPS injected intraperitoneally does not result in paralysis or fatality $(15,59)$. In addition, Sirén et al (58) stated that no paralysis or fatality resulted from BCG/LPS treatment when the time interval between BCG vaccination and LPS injection was $<1$ or $>4$ weeks. All these findings suggest that different dosages and protocols of BCG/LPS treatment may lead to varying effects on neurobehavior.

BCG is administered worldwide to human infants who may then suffer more or less during their later life from bacterial infection, including with LPS-producing bacteria. Therefore, there are numerous individuals who receive neonatal BCG immunization and suffer LPS exposure in adulthood. Mice in the present study also experienced neonatal BCG immunization and LPS-challenge in adulthood, meaning the model used in the present study may be comparable to humans. Furthermore, the BCG used in the present study is identical to the vaccine administered to humans and the age, dosage and route of vaccination in the present study are also similar to those for humans. Therefore, it is worth investigating if neonatal BCG vaccination exerts similar effects on brain development and behavior in humans. These results may not be limited to infection with LPS/LPS-producing bacteria. The present study provides a basis for further examination and provides a useful animal model for investigating the neurobehavioral effects of physiological neonatal immune activation.

In conclusion, the neonatal BCG vaccination alleviated the neurobehavioral impairments and neuroinflammation induced by exposure of adult mice to LPS. The results of the present study reveal the protective effect of BCG on the CNS following exposure to LPS, and encourage further study to investigate the use of immunoregulatory therapy for the treatment of neuropsychiatric disorders.

\section{Acknowledgements}

The authors would like to thank Dr. Zejie Zuo, Dr. Yingying Wu and Ms. Yunlong Xu (SYSU) for their invaluable comments. The authors would also like to thank Technician Qunfang Yuan (SYSU) for her technical instruction. The present study was supported by the National Natural Science Foundation of China (grant no. 31371130), the Special Foundation of Education Department of Guangdong Province (grant no. 2010-036) and the Medical Scientific Research Foundation of Guangdong Province, China (grant no. 2013-159).

\section{References}

1. Centers for Disease Control and Prevention (CDC): Global routine vaccination coverage-2012. MMWR Morb Mortal Wkly Rep 62: 858-861, 2013.
2. Gandhi NM,Morales A and Lamm DL: Bacillus Calmette-Guérin immunotherapy for genitourinary cancer. BJU Int 112: 288-297, 2013.

3. Petrovsky N: Immunomodulation with microbial vaccines to prevent type 1 diabetes mellitus. Nat Rev Endocrinol 6: 131-138, 2010.

4. Stewart JH IV and Levine EA: Role of bacillus Calmette-Guérin in the treatment of advanced melanoma. Expert Rev Anticancer Ther 11: 1671-1676, 2011.

5. Ristori G, Romano S, Cannoni S, Visconti A, Tinelli E, Mendozzi L, Cecconi P, Lanzillo R, Quarantelli M, Buttinelli C, et al: Effects of Bacille Calmette-Guerin after the first demyelinating event in the CNS. Neurology 82: 41-48, 2014.

6. Laćan G, Dang H, Middleton B, Horwitz MA, Tian J, Melega WP and Kaufman DL: Bacillus Calmette-Guerin vaccine-mediated neuroprotection is associated with regulatory $\mathrm{T}$-cell induction in the 1-methyl-4-phenyl-1,2,3,6-tetrahydropyridine mouse model of Parkinson's disease. J Neurosci Res 91: 1292-1302, 2013.

7. Lee J, Reinke EK, Zozulya AL, Sandor M and Fabry Z: Mycobacterium bovis bacille Calmette-Guerin infection in the CNS suppresses experimental autoimmune encephalomyelitis and Th17 responses in an IFN-gamma-independent manner. J Immunol 181: 6201-6212, 2008.

8. Bilbo SD and Schwarz JM: Early-life programming of later-life brain and behavior: A critical role for the immune system. Front Behav Neurosci 3: 14, 2009.

9. Spencer SJ, Heida JG and Pittman QJ: Early life immune challenge-effects on behavioural indices of adult rat fear and anxiety. Behav Brain Res 164: 231-238, 2005.

10. Bilbo SD and Schwarz JM: The immune system and developmental programming of brain and behavior. Front Neuroendocrinol 33: 267-286, 2012.

11. Bitanihirwe BK, Peleg-Raibstein D, Mouttet F, Feldon J and Meyer U: Late prenatal immune activation in mice leads to behavioral and neurochemical abnormalities relevant to the negative symptoms of schizophrenia. Neuropsychopharmacology 35: 2462-2478, 2010.

12. Winter C, Djodari-Irani A, Sohr R, Morgenstern R, Feldon J, Juckel G and Meyer U: Prenatal immune activation leads to multiple changes in basal neurotransmitter levels in the adult brain: Implications for brain disorders of neurodevelopmental origin such as schizophrenia. Int J Neuropsychopharmacol 12: 513-524, 2009.

13. Bland ST, Beckley JT, Young S, Tsang V, Watkins LR, Maier SF and Bilbo SD: Enduring consequences of early-life infection on glial and neural cell genesis within cognitive regions of the brain. Brain Behav Immun 24: 329-338, 2010.

14. Bilbo SD, Barrientos RM, Eads AS, Northcutt A, Watkins LR, Rudy JW and Maier SF: Early-life infection leads to altered BDNF and IL-1beta mRNA expression in rat hippocampus following learning in adulthood. Brain Behav Immun 22: 451-455, 2008.

15. Henry CJ, Huang Y, Wynne A, Hanke M, Himler J, Bailey MT, Sheridan JF and Godbout JP: Minocycline attenuates lipopolysaccharide (LPS)-induced neuroinflammation, sickness behavior, and anhedonia. J Neuroinflammation 5: 15, 2008.

16. Rostami F, Oryan S, Ahmadiani A and Dargahi L: Morphine preconditioning protects against LPS-induced neuroinflammation and memory deficit. J Mol Neurosci 48: 22-34, 2012.

17. Bilbo SD, Levkoff LH, Mahoney JH, Watkins LR, Rudy JW and Maier SF: Neonatal infection induces memory impairments following an immune challenge in adulthood. Behav Neurosci 119: 293-301, 2005.

18. Bilbo SD, Biedenkapp JC, Der-Avakian A, Watkins LR, Rudy JW and Maier SF: Neonatal infection-induced memory impairment after lipopolysaccharide in adulthood is prevented via caspase-1 inhibition. J Neurosci 25: 8000-8009, 2005.

19. Hollands C: The animals (scientific procedures) act 1986. Lancet 2: 32-33, 1986.

20. Shen H, Huang H, Wang J, Ye S, Li W, Wang K, Zhang G and Wang P: Neonatal vaccination with Bacillus Calmette-Guérin elicits long-term protection in mouse-allergic responses. Allergy 63: 555-563, 2008.

21. Lin S, Thomas TC, Storlien LH and Huang XF: Development of high fat diet-induced obesity and leptin resistance in C57Bl/6 J mice. Int J Obes Relat Metab Disord 24: 639-646, 2000

22. Wang YJ, Zheng YL, Lu J, Chen GQ, Wang XH, Feng J, Ruan J, Sun X, Li CX and Sun QJ: Purple sweet potato color suppresses lipopolysaccharide-induced acute inflammatory response in mouse brain. Neurochem Int 56: 424-430, 2010. 
23. Yirmiya R: Endotoxin produces a depressive-like episode in rats. Brain Res 711: 163-174, 1996.

24. Frenois F, Moreau M, O'Connor J, Lawson M, Micon C, Lestage J, Kelley KW, Dantzer R and Castanon N: Lipopolysaccharide induces delayed FosB/DeltaFosB immunostaining within the mouse extended amygdala, hippocampus and hypothalamus, that parallel the expression of depressive-like behavior. Psychoneuroendocrinology 32: 516-531, 2007.

25. Sulakhiya K, Kumar P, Gurjar SS, Barua CC and Hazarika NK Beneficial effect of honokiol on lipopolysaccharide induced anxiety-like behavior and liver damage in mice. Pharmacol Biochem Behav 132: 79-87, 2015.

26. Fujioka $\mathrm{H}$ and Akema T: Lipopolysaccharide acutely inhibits proliferation of neural precursor cells in the dentate gyrus in adult rats. Brain Res 1352: 35-42, 2010.

27. Bachstetter AD, Jernberg J, Schlunk A, Vila JL, Hudson C, Cole MJ, Shytle RD, Tan J, Sanberg PR, Sanberg CD, et al: Spirulina promotes stem cell genesis and protects against LPS induced declines in neural stem cell proliferation. PloS One 5: e10496, 2010

28. Pollmächer T, Haack M, Schuld A, Reichenberg A and Yirmiya R Low levels of circulating inflammatory cytokines-do they affect human brain functions? Brain Behav Immun 16: 525-532, 2002.

29. Konsman JP, Parnet P and Dantzer R: Cytokine-induced sickness behaviour: Mechanisms and implications. Trends Neurosci 25 154-159, 2002.

30. Zhao W, Xie W, Xiao Q, Beers DR and Appel SH: Protective effects of an anti-inflammatory cytokine, interleukin-4, on motoneuron toxicity induced by activated microglia. J Neurochem 99: 1176-1187, 2006

31. Spera PA, Ellison JA, Feuerstein GZ and Barone FC: IL-10 reduces rat brain injury following focal stroke. Neurosci Lett 251 189-192, 1998

32. Saraiva M and O'Garra A: The regulation of IL-10 production by immune cells. Nat Rev Immunol 10: 170-181, 2010.

33. Stumhofer JS, Silver JS, Laurence A, Porrett PM, Harris TH, Turka LA, Ernst M, Saris CJ, O'Shea JJ and Hunter CA: Interleukins 27 and 6 induce STAT3-mediated T cell production of interleukin 10. Nat Immunol 8: 1363-1371, 2007.

34. McGeachy MJ, Bak-Jensen KS, Chen Y, Tato CM, Blumenschein W, McClanahan T and Cua DJ: TGF-beta and IL-6 drive the production of IL-17 and IL-10 by T cells and restrain T (H)-17 cell-mediated pathology. Nat Immunol 8: 1390-1397, 2007.

35. O'Garra A, Barrat FJ, Castro AG, Vicari A and Hawrylowicz C: Strategies for use of IL-10 or its antagonists in human disease. Immunol Rev 223: 114-131, 2008.

36. Lagranderie $M$ and Guyonvarc'h PM: The interplay between bacillus Calmette-Guérin and Treg cells and its role to prevent or cure inflammatory diseases. Expert Rev Clin Immunol 10: 741-745, 2014.

37. Madura Larsen J, Benn CS, Fillie Y, van der Kleij D, Aaby P and Yazdanbakhsh M: BCG stimulated dendritic cells induce an interleukin-10 producing T-cell population with no T helper 1 or T helper 2 bias in vitro. Immunology 121: 276-282, 2007.

38. Li Q and Shen HH: Neonatal bacillus Calmette-Guérin vaccination inhibits de novo allergic inflammatory response in mice via alteration of CD4+CD25+ T-regulatory cells. Acta Pharmacol Sin 30: 125-133, 2009.

39. Kristensen I, Aaby P and Jensen $\mathrm{H}$ : Routine vaccinations and child survival: Follow up study in Guinea-Bissau, West Africa. BMJ 321: 1435-1438, 2000

40. Roth A, Gustafson P, Nhaga A, Djana Q, Poulsen A, Garly ML, Jensen H, Sodemann M, Rodriques A and Aaby P: BCG vaccination scar associated with better childhood survival in Guinea-Bissau. Int J Epidemiol 34: 540-547, 2005

41. Martino A, Sacchi A, Sanarico N, Spadaro F, Ramoni C, Ciaramella A, Pucillo LP, Colizzi V and Vendetti S: Dendritic cells derived from BCG-infected precursors induce Th2-like immune response. J Leukoc Biol 76: 827-834, 2004.

42. Stepanichev M, Dygalo NN, Grigoryan G, Shishkina GT and Gulyaeva N: Rodent models of depression: Neurotrophic and neuroinflammatory biomarkers. Biomed Res Int 2014: 932757, 2014.
43. Godbout JP, Moreau M, Lestage J, Chen J, Sparkman NL, O'Connor J, Castanon N, Kelley KW, Dantzer R and Johnson RW: Aging exacerbates depressive-like behavior in mice in response to activation of the peripheral innate immune system. Neuropsychopharmacology 33: 2341-2351, 2008

44. Pariante CM and Lightman SL: The HPA axis in major depression: Classical theories and new developments. Trends Neurosci 31: 464-468, 2008.

45. Xu Q, Yi LT, Pan Y, Wang X, Li YC, Li JM, Wang CP and Kong LD: Antidepressant-like effects of the mixture of honokiol and magnolol from the barks of Magnolia officinalis in stressed rodents. Prog Neuropsychopharmacol Biol Psychiatry 32: $715-725,2008$

46. Munhoz CD, Sorrells SF, Caso JR, Scavone C and Sapolsky RM: Glucocorticoids exacerbate lipopolysaccharide-induced signaling in the frontal cortex and hippocampus in a dose-dependent manner. J Neurosci 30: 13690-13698, 2010.

47. Banks WA: Blood-brain barrier transport of cytokines: A mechanism for neuropathology. Curr Pharm Des 11: 973-984, 2005.

48. Schwartz M and Shechter R: Systemic inflammatory cells fight off neurodegenerative disease. Nat Rev Neurol 6: 405-410, 2010.

49. Yirmiya R and Goshen I: Immune modulation of learning, memory, neural plasticity and neurogenesis. Brain Behav Immun 25: 181-213, 2011.

50. Combrinck MI, Perry VH and Cunningham C: Peripheral infection evokes exaggerated sickness behaviour in pre-clinical murine prion disease. Neuroscience 112: 7-11, 2002.

51. Leentjens J, Kox M, Stokman R, Gerretsen J, Diavatopoulos DA, van Crevel R, Rimmelzwaan GF, Pickkers P and Netea MG: BCG vaccination enhances the immunogenicity of subsequent influenza vaccination in healthy volunteers: A randomized, placebo-controlled pilot study. J Infect Dis 212: 1930-1938, 2015.

52. Midgley CM, Bajwa-Joseph M, Vasanawathana S, Limpitikul W, Wills B, Flanagan A, Waiyaiya E, Tran HB, Cowper AE, Chotiyarnwong P, et al: An in-depth analysis of original antigenic sin in dengue virus infection. J Virol 85: 410-421, 2011.

53. Kelley KW, O'Connor JC, Lawson MA, Dantzer R, Rodriguez-Zas SL and McCusker RH: Aging leads to prolonged duration of inflammation-induced depression-like behavior caused by Bacillus Calmette-Guérin. Brain Behav Immun 32: 63-69, 2013.

54. Moreau M, André C, O'Connor JC, Dumich SA, Woods JA, Kelley KW, Dantzer R, Lestage J and Castanon N: Inoculation of Bacillus Calmette-Guerin to mice induces an acute episode of sickness behavior followed by chronic depressive-like behavior. Brain Behav Immun 22: 1087-1095, 2008.

55. Vijaya Kumar K, Rudra A, Sreedhara MV, Siva Subramani T, Prasad DS, Das ML, Murugesan S, Yadav R, Trivedi RK, Louis JV, et al: Bacillus Calmette-Guérin vaccine induces a selective serotonin reuptake inhibitor (SSRI)-resistant depression like phenotype in mice. Brain Behav Immun 42: 204-211, 2014.

56. Dantzer R, O'Connor JC, Lawson MA and Kelley KW: Inflammation-associated depression: From serotonin to kynurenine. Psychoneuroendocrinology 36: 426-436, 2011.

57. O'Connor JC, Andrú C, Wang Y, Lawson MA, Szegedi SS, Lestage J, Castanon N, Kelley KW and Dantzer R: Interferon-gamma and tumor necrosis factor-alpha mediate the upregulation of indoleamine 2,3-dioxygenase and the induction of depressive-like behavior in mice in response to bacillus Calmette-Guerin. J Neurosci 29: 4200-4209, 2009.

58. Siren AL, McCarron R, Wang L, Garcia-Pinto P, Ruetzler C, Martin D and Hallenbeck JM: Proinflammatory cytokine expression contributes to brain injury provoked by chronic monocyte activation. Mol Med 7: 219-229, 2001.

59. Sekio $M$ and Seki $K$ : Lipopolysaccharide-induced depressive-like behavior is associated with $\alpha_{1}$-adrenoceptor dependent downregulation of the membrane GluR 1 subunit in the mouse medial prefrontal cortex and ventral tegmental area. Int J Neuropsychopharmacol 18: 18, 2014. 